\title{
Effect of Yoga Therapy on Low Back Pain Management Among Older Adults: Implications for Gerontology Counselling
}

\author{
Joy I. Anyanwu ${ }^{1}$, Oliver Rotachukwu Ngwoke ${ }^{2}$, Vera Victor-Aigbodion ${ }^{1}$, \\ Ogechi Nnamani ${ }^{1} \&$ Bartholomew C. Nwefuru ${ }^{1}$ \\ ${ }^{1}$ Department of Educational Foundations, University of Nigeria, Nsukka, Nigeria \\ ${ }^{2}$ Department of Human Kinetics and Health Education, University of Nigeria, Nsukka, Nigeria \\ Correspondence: Oliver Rotachukwu Ngwoke, Department of Human Kinetics and Health Education, University \\ of Nigeria, Nsukka, Nigeria. E-mail: oliver.ngwoke@unn.edu.ng
}

Received: May 12, 2019 Accepted: June 15, 2019 Online Published: June 20, 2019

doi:10.5539/gjhs.v11n8p1 URL: https://doi.org/10.5539/gjhs.v11n8p1

\begin{abstract}
Objective: This research aimed to determine the effect of yoga therapy in managing low back pain (LBP) among older adults.

Method: 40 participants who were having low back pain were assessed. All participants completed baseline evaluation before beginning the Yoga intervention and at 6,12 and 18 weeks. Participants completed a questionnaire titled Oswestry Low Back Pain Disability Questionnaire (ODQ). The statistical tool used for data analysis was within-and-between subjects ANOVA.

Results: The finding showed no significant difference in the baseline assessment for LBP between the treatment group and the waitlisted control group, $F(1,38)=2.697, P=.000, \eta^{2}=.066$. The posttest assessment at 6 th week revealed a significant reduction of LBP among older adults in the yoga treatment group compared with those in the waitlisted control group, $F(1,37)=3209.376, P=.000, \eta^{2}=.989$. The assessment at 12 th week revealed significant reduction in LBP among older adult in the yoga treatment group compared with those in the waitlisted control group, $F(1,36)=2389.154, P=.000, \eta^{2}=.985$. The assessment at 18 th week further revealed a significant reduction in LBP among older adult in the yoga treatment group compared with those in the waitlisted control group, $F(1,36)=2775.162, P=.000, \eta^{2}=.987$.
\end{abstract}

Conclusion: Yoga therapy is an effective intervention for managing low back pain among older adults. Thus, gerontology counsellors can provide help to older people with low back pain within the framework of Yoga therapy. Further studies are required to find out and corroborate the efficacy of Yoga Therapy in managing low back pain among older adults.

Keywords: Yoga Therapy, low back pain, management, older adults and gerontologycounselling

\section{Introduction}

Low back pain (LBP) is seen as one of the main immobilizing health challenges among older people ranging from 60 and above resulting in painful conditions and disability (Bain et al., 2015). LBP has no definite history but from different pain sources (Middleton \& Fish, 2009). Heneweer, Picavet, Staes, Kiers and Vanhees (2012) were of the views that work exposures to all human body such as lifting, twisting, bending, and stooping leads to body vibrations are potential risk factors for FBP in the older adults working age. According to Cypress (1983), people aged 60 years and above are topping the most common people suffering from LBP. Leopoldino et al. (2016) reported that LBP prevalence in community-dwelling older adults in one year ranges from 13 to $50 \%$ globally. Hides, Jull and Richardson (2001) reported that over $70 \%$ of the human population is expected to experience LBP at some point in time in their lifetime and recurrence rate can be as high as $85 \%$. Tarzian and Hoffmann (2005) also report that $80 \%$ of older adults experience musculoskeletal pain with the majority attributed to LBP. Accordingly, the scholars maintained that low back pain among older adults is underreported globally while patients are treated inadequately which contributes to the high prevalence cases of LBP among older people of the society. Balague and Pellisé (2016) opined that LBP starts in an individual's life as early as teenage and progressively expands at 60 years and above. Dijken, Fjellman-Wiklund and Hildingsson (2008) were of the views that LBP are attributed to occupational exposure among working age. Palacios-Ceña et al. (2015) reported that LBP is a common illness 
among older adults of retirement age. Thomas, Peat, Harris, Wilkie and Croft (2004) identified severe and chronic LBP among older adults of working age. Docking et al. (2006) reported that back pain (BP) prevalence in one-month changes from 3.8\% between older adults of 77-79 years to $9.7 \%$ between those at $90-100$ years old. Williams et al. (2015) admitted that severe LBP is common among people of 80 years and above than those at the $50 s^{\prime}$. The authors further noted that sometimes severe LBP are poorly handled which may generate to individual functional disabilities. In the other hand, Hartvigsen, Frederiksen and Christensen (2005) reported that chronic LBP was common among older people aged 65 and above and last for a duration of three months. Lavsky-Shulan, et al. (1985) were of the views that chronic LBP illness is more occurring among people in declining age and also common amongst female than male counterparts. Jacobs, Hammerman-Rozenberg, Cohen, and Stessman (2006) reported that women hypertension, joint pain, pre-existing LBP, and loneliness, were ascribed for developing persistent LBP in older adults aged 70 years. Williams, et al. (2015) revealed that comorbid chronic conditions were positively related to at least one LBP episode in the last month in low- and middle-income nations. Furthermore, the authors restated that the odds of LBP were 2.7 times higher among adults with one chronic comorbid condition, compared to adults without comorbidities, while the odds ratio was 4.8 for an individual with two or more comorbidities. Hammill, Beazell and Hart (2008) asserted that chronic LBP interferes with individual daily activities increasing death rate, reducing work performance, and poorly impacting self-concern. Chronic LBP has both emotional and psychological consequence and proves difficult to be cured as $17 \%$ death rate are ascribed to chronic LBP (Hammill, Beazell, \& Hart, 2008).

The advancement of medicine has not only contributed to healthy living but has positively expanded human lifespan (Tse, Pun, \& Benzie, 2005). Luo, Pietrobon, Sun, Liu and Hey (2004) were of the opinion that life threat mounted on humans especially the older adults had made them turn to some supplementary and alternative medicine in order to address their illness. Barnes and Bloom (2008) asserted that Yoga is the type complementary and alternative medicine that includes different practices such as physical posture, breathing exercise and medication. Barnes and Bloom (2008) opined that Yoga has been in practice among older American adults suffering from LBP since 2007. Yoga can be traced to ancient India. It is the practice of the inner mind (Saper, 2004). Hayes (2010) reported that yoga practice has numerous styles and characteristics by the combination of physical poses (asanas), controlled breathing (pranayama) and consequently the integration of meditation (dhyana) NHIS (2007) were of the view that the application of yoga in the USA increased between 2002 to 2007. As of 2007 over 13 million older adults had applied yoga (Barnes, 2008; Birdee et al., 2008). NHIS (2012) further reported that the application of yoga in the USA increased in 2012 to over 21 million older adults (Cramer et al., 2016). On the significant role of yoga, the International Association of Yoga Therapists (2016) recommends people seeking to manage their health issues adequately should always visit yoga therapists. According to this Association, therapeutic yoga is the application of yoga to solve or manage older adults' LBP and other related health conditions. Bussing, Ostermann, Ludtke and Michalsen (2012) stated that yoga helps in managing pain and disability conditions such as back pain. This seems to validate the position of NHIS in 2002 according to Birdee et al. (2008) on the alternative medicine supplement survey wherein over 10 million USA adults described using yoga for health reasons; $10.5 \%$ of yoga users said that their use was for musculoskeletal conditions and $76 \%$ of these users reported that the yoga was useful. Cramer, et al. (2016) reporting about NHIS' 2012 study, noted that $19.7 \%$ of yoga users said their use was specifically for back pain. Wren (2011) also pointed out that yoga therapy is efficient in persistent pain conditions, such as changes in physiological, psychological and behavioural factors. Yoga as one of the complementary and alternative medicine is used to treat LBP (Wolsko, 2003).

\section{Methods}

\subsection{Study Design and Ethical Considerations}

The study used a group randomized controlled trial design. The study protocol was approved by the research ethics committee of the Faculty of Education, University of Nigeria Nsukka.

\subsection{Study Area and Participants}

The study was carried out in South-East, Nigeria. Older adults within the study area were screened by the gerontology counsellors. The study participants were older adults with low back pain that had not received any treatment, besides symptomatic medication. Forty (40) eligible patients aged 50years and above, with LBP for more than a year, who agreed to provide informed consent were included in the study. The 40 eligible participants were randomized into one of two study groups: yoga intervention group $(n=20)$ and waitlist control group $(n=20)$ using computer-generated random numbers. 


\subsection{Measures}

Participants completed a questionnaire measuring LBP, titled Oswestry Low Back Pain Disability Questionnaire (ODQ, Fairbank, \& Pynsent, 2000) at baseline, 6, 12, and 18 weeks of yoga therapy. The ODQ which comprises of 10 items arranging from no impairment (0) to maximum impairment (5) which measure disability due to LBP. In the ODQ, one point is given for each item checked.

\subsection{Intervention}

The yoga session activities included relaxation, breathing exercise, physical posture, guided meditation, and chants. The intervention incorporated cognitive-behavioural and mindfulness components. Yoga exercises and cognitive-behavioural components were further derived from previous intervention by Kalsa et al. (2015). The study participants were also given a self-help guide for home practices which complemented the in-session yoga activities.

\subsection{Procedure}

Based on the set inclusionary-exclusionary criteria, gerontology counsellors referred eligible participants for yoga therapy. The study protocol was described to all participants, and informed consent forms were completed after an adequate description of the research objective, methodology and potential harms. After completing the baseline evaluation, the participants were exposed to 18 -weeks yoga intervention sessions. The study participants were asked to attend the group intervention session twice weekly. Each yoga session lasted for 90 minutes. All waitlisted participants were scheduled to start the yoga sessions straight away when the yoga group participants have had their last yoga session and assessment. Participants in the waitlist group had casual meetings and discussions about LBP management during the waiting period.

\subsection{Statistical Data Analysis}

The statistical tool used for data analysis was within-and-between subjects ANOVA. Data were normally distributed and there were no missing data. All analyses were conducted using SPSS computer software, version 22.0 (IBM Corp. NY, United States). All p-values $\leq 0.05$ were regarded as significant.

\section{Results}

There are twenty (20) participants in the treatment group (10males, 10females) and twenty (20) waitlisted controlled group participants (10 males, 10 females). Table 1 shows the descriptive statistics by study groups.

Table 1. Descriptive statistics by study groups

\begin{tabular}{|c|c|c|c|c|c|}
\hline Group & & Baseline & $6^{\text {th }}$ Week & $12^{\text {th }}$ Week & $18^{\text {th }}$ Week \\
\hline \multirow{4}{*}{ Yoga intervention group } & $\mathrm{M}$ & 82.15 & 5.70 & 4.35 & 4.35 \\
\hline & $\mathrm{N}$ & 20 & 19 & 18 & 20 \\
\hline & SD & 5.56 & 2.56 & 3.77 & 3.78 \\
\hline & SEM & 1.24 & .57 & .84 & .84 \\
\hline \multirow{4}{*}{ Waitlisted control group } & $\mathrm{M}$ & 79.40 & 79.27 & 79.37 & 78.84 \\
\hline & $\mathrm{N}$ & 20 & 19 & 19 & 19 \\
\hline & SD & 5.02 & 5.18 & 5.11 & 5.60 \\
\hline & SEM & 1.12 & 1.19 & 1.28 & 1.17 \\
\hline
\end{tabular}


Table 2. ANOVA statistics indicating the effect of yoga therapy in managing low back pain (LBP) among older adults

\begin{tabular}{|c|c|c|c|c|c|c|c|c|}
\hline & & & Sum of Squares & $\mathrm{df}$ & Mean Square & $\mathrm{F}$ & Sig. & Eta Squared \\
\hline & Between Groups & (Combined) & 75.625 & 1 & 75.625 & & & \\
\hline \multirow[t]{3}{*}{ Baseline } & Within Groups & & 1065.350 & 38 & 28.036 & 2.697 & .109 & .066 \\
\hline & Total & & 1140.975 & 39 & & & & \\
\hline & Between Groups & (Combined) & 52727.808 & 1 & 52727.808 & & & \\
\hline \multirow[t]{3}{*}{$6^{\text {th }}$ Week } & Within Groups & & 607.884 & 37 & 16.429 & 3209.376 & .000 & .989 \\
\hline & Total & & 53335.692 & 38 & & & & \\
\hline & Between Groups & (Combined) & 53029.266 & 1 & 53029.266 & & & \\
\hline \multirow[t]{3}{*}{$12^{\text {th }}$ Week } & Within Groups & & 799.050 & 36 & 22.196 & 2389.154 & .000 & .985 \\
\hline & Total & & 53828.316 & 37 & & & & \\
\hline & Between Groups & (Combined) & 53819.734 & 1 & 53819.734 & & & \\
\hline \multirow[t]{2}{*}{$18^{\text {th }}$ Week } & Within Groups & & 698.161 & 36 & 19.393 & 2775.162 & .000 & .987 \\
\hline & Total & & 54517.895 & 37 & & & & \\
\hline
\end{tabular}

As shown in Table 2, the finding showed no significant difference in the baseline assessment for LBP between the treatment group $(82.15 \pm 5.56)$ and the waitlisted control group $(79.40 \pm 5.02), F(1,38)=2.697, P=.000, \eta^{2}=.066$ The posttest assessment at $6^{\text {th }}$ week revealed a significant reduction of LBP among older adults in the yoga treatment group (5.70 \pm 2.56$)$ compared with those in the waitlisted control group $(79.27 \pm 5.18), F(1,37)=$ $3209.376, P=.000, \eta^{2}=.989$.

The assessment at $12^{\text {th }}$ week revealed significant reduction in LBP among older adult in the yoga treatment group (4.35 \pm 3.77$)$ compared with those in the waitlisted control group $(79.37 \pm 5.11), F(1,36)=2389.154, P=.000$, $\eta^{2}=985$. The assessment at $18^{\text {th }}$ week further revealed significant reduction in LBP among older adult in the yoga treatment group $(4.35+3.78)$ compared with those in the waitlisted control group $(78.84 \pm 5.60), F(1,36)=2775.162$, $P=.000, \eta^{2}=.987$.

\section{Discussion}

This study showed that yoga therapy was effective in reducing LBP among older adults. The yoga therapy intervention group result showed that older adults with LBP can be effectively managed through the application of yoga therapy by gerontology counsellors. The position was justified with the result of those in this group when compared to their counterpart in the waitlisted controlled group after 4 times administration of the test. The finding of this study is consistent with that of Posadzki, and Ernst (2011) who found that yoga therapy leads to a significantly greater reduction in low back pain than usual care, education or conventional therapeutic exercises. Büssing, Poier, Désirée, Thomas and Michalsen (2017) reported that yoga intervention has significant effects in treating patients with low back pain. Kelly (2009) found that yoga is very much efficient in the treatment of LBP. In a study by Sabet, Ganji and Dehghani (2017) on the effect of 8 weeks of yoga selected exercises on pain and functional disability in women with non-specific chronic low back pain, it was reported that treatment of LBP can be done with the use of yoga. Combs and Thorn (2015) found that proper management of yoga enables easy treatment of LBP. Combs and Thorn (2014) reported that the use of yoga was efficacious in treating chronic low back pain among individuals of different category. Tse, Pun and Benzie, (2005) reported that yoga has not only contributed to healthy living but has positively expanded human lifespan. Accordingly, the International Association of Yoga Therapists (2016) also recommends that management of health issues especially LBP can be better managed with the use of yoga therapy. McCall (2007) found that yoga helps in managing pain and disability conditions such as back pain. The finding also validates the position of NHIS in 2002 as articulated by Birdee et al. (2008), that yoga is an alternative medicine for health reasons. Wren (2011) found that yoga therapy is efficient in persistent pain conditions, such as changes in physiological, psychological and behavioural factors. Birdee et al. (2008) noted that yoga is a complementary and alternative medicine used to treat LBP. Madanmohan, Udupa, Bhavanani, Shatapathy and Sahai (2004) reported that yoga can be effectively used in the treatment of cardiovascular disorders as its use positively reflect in the reduction of systolic and diastolic blood pressure and of the heart rate as well as in higher heart rate variability. Vadiraja, Rao, Nagarathna, Nagendra, Rekha and Vanitha, 
(2009) also reported that yoga therapy intervention is essential in eliminating human diseases such as emotional risk factors like negative effect, anxiety and depression. Baldwin (1999) found that yoga practices give protective factors like positive effect. Also, Smith (2005) reported that yoga intervention programmes offer participants a state of relaxation.

\section{Conclusion}

This study evaluated the effect of yoga therapy in managing low back pain (LBP) among older adults. The yoga intervention brought about a significant reduction in LBP scores of the recipients compared to participants in a waitlisted controlled group. Thus, we concluded that yoga therapy with the help of gerontology counsellors could be an effective treatment approach for reducing LBP symptoms among older adults. To that end, further clinical evaluation is very much needed to ascertain the effect of yoga therapy for other categories of persons with LBP.

\section{Competing Interests Statement}

The authors declare that there are no competing or potential conflicts of interest.

\section{References}

Balagué, F., \& Pellisé, F. (2016). Adolescent idiopathic scoliosis and back pain.Scoliosis and Spinal Disorders. https://doi.org/10.1186/s13013-016-0086-7

Baldwin, M. C. (1999). Psychological and physiological influences of Hatha Yoga training onhealthy, exercising adults.DissAbstrInt Sect AeHumanitSocSci, 6, 4.

Barnes, P. M., \& Bloom, B. (2008).Complementary and alternative medicine use among adultsand children in the United States.Centers for Disease Control and Prevention, National Center for Health Statistics 2007, 12, 1-9. https://doi.org/10.1037/e623942009-001

Birdee, G. S., Legedza, A. T., Saper, R. B., Bertisch, S. M., Eisenberg, D. M., \& Phillips, R. S. (2008). Characteristics of yoga users: results of a national survey. Journal of General Internal Medicine, 23(10), 1653-1658. https://doi.org/10.1007/s11606-008-0735-5

Bressler, H. B., Keyes, W. J., Rochon, P. A., \& Badley, E. (1999). The prevalence of low back pain in the elderly: a $\begin{array}{lllll}\text { systematic review of the literature. Spine, } & \text { 1813-1819. }\end{array}$ https://doi.org/10.1097/00007632-199909010-00011

Bussing, A., Ostermann, T., Ludtke, R., \& Michalsen, A. (2012). Effects of yoga interventions onpain and pain-associated disability: A meta-analysis. Journal of Pain, 13(1), 1-9. https://doi.org/10.1016/j.jpain.2011.10.001

Büssing, A., Poier, D., Ostermann, T., Kröz, M., \& Michalsen, A. (2017). Treatment of Chronic Lower Back Pain: Study Protocol of a Comparative Effectiveness Study on Yoga, Eurythmy Therapy, and Physiotherapeutic Exercises. Complementary Medicine Research, 25. https://doi.org/10.1159/000471801

Combs, T. (2014). Barriers and facilitators to yoga use in a population of individuals with self-reported chronic low back pain: A qualitative approach. Complementary Therapies in Clinical Practice. 20. https://doi.org/10.1016/j.ctcp.2014.07.006

Combs, T. (2015). Yoga Attitudes in Chronic Low Back Pain: Roles of Catastrophizingand Fear of Movement. Complementary Therapies in Clinical Practice. 21. https://doi.org/10.1016/j.ctcp.2015.06.006

Cramer, H., Ward, L., Steel, A., Lauche, R., Dobos, G., \& Zhang, Y. (2016). Prevalence, patterns, and predictors of yoga use: results of a U.S. nationally representative survey. American Journal of Preventive Medicine, 50(2), 230-235.https://doi.org/10.1016/j.amepre.2015.07.037

Cypress, B. K. (1983). Characteristics of physician visits for back symptoms: a national perspective. Am J Public Health, 73, 389-95.https://doi.org/10.2105/AJPH.73.4.389

Dijken, C.B-V, Fjellman-Wiklund, A., \& Hildingsson, C. (2008). Low back pain, lifestyle factors and physical activity: a population-based study. J Rehabil Med., 40, 864-869. https://doi.org/10.2340/16501977-0273

Docking, R.E., Fleming, J., Brayne, C., Zhao, J., Macfarlane, G.J., \& Jones, G.T. (2011). Epidemiology of back pain in older adults: prevalence and risk factors for back pain onset. Rheumatology, 50, 1645-53. https://doi.org/10.1093/rheumatology/ker175

Fairbank, J. C. T., \& Pynsent, P. B. (2000). The Oswestry Low Back Pain Disability Questionnaire. Spine, 25(22), 2940-2953. https://doi.org/10.1097/00007632-200011150-00017 
Hammill, R. R., Beazell, J. R., \& Hart, J. M. (2008).Neuromuscular consequences of low backpain and core dysfunction.Clin Sports Med, 27, 449-462.https://doi.org/10.1016/j.csm.2008.02.005

Hartvigsen, J., Frederiksen, H., \& Christensen, K. (2005). Back and neck pain in seniors-prevalence and impact. Eur Spine J., 15, 802-6. https://doi.org/10.1007/s00586-005-0983-6

Hayes, M., \& Chase, S. (2010).Prescribing yoga. Primary Care: Clinics in Office Practice, 37, 31-47. https://doi.org/10.1016/j.pop.2009.09.009

Heneweer, H., Picavet, H. S. J., Staes, F., Kiers, H., \& Vanhees, L. (2012). Physical fitness, rather than self-reported physical activities, is more strongly associated with low back pain: evidence from a working population. Eur Spine J., 21, 1265-72. https://doi.org/10.1007/s00586-011-2097-7

Hides, J. A., Jull, G. A., \& Richardson, C. A. (2001). Long-term effects of specific stabilizing exercises for rest-episode low back pain. Spine (Phila Pa 1976), 26, 243-248. https://doi.org/10.1097/00007632-200106010-00004

International Association of Yoga Therapists (IAYT). (2016). Contemporary definitions of yoga therapy. Retrieved from http://www.iayt.org/?page=ContemporaryDefinition

Jacobs, J. M., Hammerman-Rozenberg, R., Cohen, A., \& Stessman, J. (2006). Chronic back pain among the elderly: prevalence, associations, and predictors. Spine. 31, E203-7. https://doi.org/10.1097/01.brs.0000206367.57918.3c

Khalsa, M. K., Greiner-Ferris, J. M., Hofmann, S. G., \& Khalsa, S. B. (2015). Yoga-enhanced cognitive behavioural therapy (Y-CBT) for anxiety management: a pilot study. Clinical Psychology \& Psychotherapy, 22(4), 364-371. https://doi.org/10.1002/cpp.1902

Kelly, Z. (2009). Is yoga an effective treatment for low back pain: A research review. International Journal of Yoga Therapy, 19, 103-112.

Lavsky-Shulan, M., Wallace, R. B., Kohout, F. J., Lemke, J. H., Morris, M. C., \& Smith, I. M. (1985) Prevalence and functional correlates of low back pain in the elderly: the Iowa 65+ Rural Health Study. J Am GeriatrSoc, 33, 23-28. https://doi.org/10.1111/j.1532-5415.1985.tb02855.x

Leopoldino, A. A. O., Diz, J. B. M., Martins, V. T., Henschke, N., Pereira, L. S. M., Dias, R. C., \& Oliveira, V. C. (2016). Prevalence of low back pain in older Brazilians: A systematic review with meta-analysis. Rev Bras Reumatol Engl Ed, 56, 258-69. https://doi.org/10.1016/j.rbr.2016.01.004

Luo, X., Pietrobon, R., Sun, S. X, Liu, G. G. \& Hey, L. (2004). Estimates and patterns of direct health care expenditures among individuals with back pain in the United States. Spine, 29, 79-86. https://doi.org/10.1097/01.BRS.0000105527.13866.0F

Madanmohan, B. A., Udupa, K., Bhavanani, A. B., Shatapathy, C. C., \& Sahai, A. (2004).Modulation of cardiovascular response to exercise by yoga training. Indian J PhysiolPharmacol, 48, 461e5.

McCall, T. (2007). Yoga as Medicine. New York: Bantam Dell.

Palacios-Ceña, D., Alonso-Blanco, C., Hernández-Barrera, V., Carrasco-Garrido, P., Jiménez-García, R., \& Fernández-de-las-Peñas, C. (2015). Prevalence of neck and low back pain in community-dwelling adults in Spain: an updated population-based national study. Eur Spine J., 24, 482-92. https://doi.org/10.1007/s00586-014-3567-5

Posadzki, P., \& Ernst, E. (2011). Yoga for low back pain: A systematic review of randomizedclinical trials. Clinical Rheumatology, 30, 1257-62. https://doi.org/10.1007/s10067-011-1764-8

Sabet, G., \& Dehghani. (2017). The effect of 8 weeks of yoga selected exercises on pain and functional disability in Women with non-specific chronic low back pain. Journal of Sport Rehabilitation, 5.25-37.

Saper, R. B., Eisenberg, D. M., Davis, R. B., Culpepper, L., \& Phillips, R. S. (2004). Prevalence and patterns of adult yoga use in the United States: results of a national survey. Alternative Therapies in Health and Medicine, $10(2), 44-9$.

Smith, J. C. (2005). Relaxation, meditation, and mindfulness: a mental health professional's guide to new and traditional approaches. New York: Springer.

Tarzian, A. J., \& Hoffmann, D. E. (2005). Barriers to managing pain in the nursing home: findings from a statewide survey. J Am Med DirAssoc, 6, S13-9. https://doi.org/10.1016/j.jamda.2005.03.016 
Thomas, E., Peat, G., Harris, L., Wilkie, R., \& Croft, P. R. (2004). The prevalence of pain and pain interference in a general population of older adults: Cross-sectional findings from the North Staffordshire Osteoarthritis Project (NorStOP). Pain, 110, 361-8. https://doi.org/10.1016/j.pain.2004.04.017

Tse, M. M. Y., Pun, S. P. Y., \& Benzie, I. F. F. (2005). Pain relief strategies used by older people with chronic pain: an exploratory survey for planning patient-centred intervention. $J$ Clin Nurs., 14, 315-20. https://doi.org/10.1111/j.1365-2702.2004.00976.x

Vadiraja, H. S., Rao, M. R., Nagarathna, R., Nagendra, H. R., Rekha, M., Vanitha, N., .. \& Rao, N. (2009). Effects of yoga program on quality of life and affect in early breast cancer patients undergoing adjuvant radiotherapy: a randomized controlled trial. Complement Ther Med, 17, $274 \mathrm{e} 80$. https://doi.org/10.1016/j.ctim.2009.06.004

Williams, J. S., Ng, N., Peltzer, K., Yawson, A., Biritwum, R., Maximova T, ... Chatterji, S. (2015). Riskfactors and disability associated with low back pain in older adults in low- and middle-income countries. Results from the WHO Study on Global AGEing and Adult Health (SAGE). PLoS ONE, 10, e0127880. https://doi.org/10.1371/journal.pone.0127880

Wolsko, P. M., Eisenberg, D. M., Davis, R. B., Kessler, R., \& Phillips, R. S. (2003). Patterns and perceptions of care for treatment of back and neck pain: results of a national survey. Spine, 28(3), 292-7.https://doi.org/10.1097/01.BRS.0000042225.88095.7C

Wren, A. A., Wright, M. A., Carson, J. W., \& Keefe, F. J. (2011). Yoga for persistent pain: new findings and directions for an ancient practice. Pain, 152(3), 477-80.https://doi.org/10.1016/j.pain.2010.11.017

\section{Copyrights}

Copyright for this article is retained by the author(s), with first publication rights granted to the journal.

This is an open-access article distributed under the terms and conditions of the Creative Commons Attribution license (http://creativecommons.org/licenses/by/4.0/). 